\title{
The Contribution of Untreated and Treated Anxiety and Depression to Prenatal, Intrapartum, and Neonatal Outcomes
}

\author{
Dotun Ogunyemi, $\mathrm{MD}^{1,2}$ Andrew Jovanovski, MD ${ }^{2}$ James Liu, MD ${ }^{1,2}$ Perry Friedman, MD ${ }^{1,2}$ \\ Nathaniel Sugiyama, MD $^{3}$ James Creps ${ }^{2}$ Ichchha Madan, MD $^{1,2}$ \\ ${ }^{1}$ Division of Maternal-Fetal Medicine, Department of Obstetrics and \\ Gynecology, Beaumont Hospital, Royal Oak, Michigan \\ 2 Department of Obstetrics and Gynecology, Oakland University, \\ William Beaumont School of Medicine, Rochester Hills, Michigan \\ ${ }^{3}$ University of Vermont Robert Larner College of Medicine, \\ Burlington, Vermont \\ Address for correspondence Dotun Ogunyemi, MD, System Vice \\ Chair of Patient Safety and Quality, Beaumont Health, MFM Fellowship \\ Director, Beaumont Hospital, Royal Oak, Professor, Oakland \\ University, William Beaumont School of Medicine, \\ 3601 West 13 mile rd. Royal Oak, MI 48073 \\ (e-mail: dogunye@outlook.com).
}

Am J Perinatol Rep 2018;8:e146-e157.

\begin{abstract}
Keywords

- anxiety

- depression

- psychotropic medication

- maternal outcome

- neonatal outcome

- neonatal adaptation syndrome

Objective To determine independent perinatal associations of anxiety and depression in women who were and were not treated with psychotropic drugs in comparison to unaffected pregnancies.

Study Design From 2013 to 2014, 978 (6.3\%) cases of anxiety/depression, of which $35 \%$ used psychotropic drugs, were compared with 14,514 (93.7\%) unaffected pregnancies using logistic regression.

Results Subjects were more likely to be Non-Hispanic Whites, use tobacco and illegal substances, be unmarried, use public insurance, and have medical complications of pregnancy. For independent maternal outcomes, untreated anxiety/depression was associated with labor induction (adjusted odds ratio $[\mathrm{aOR}]=2.02$ ), cesarean deliveries $(\mathrm{aOR}=1.69)$, longer length of stay $(\mathrm{aOR}=1.96)$, readmission $(\mathrm{aOR}=2.40)$, fever $(\mathrm{aOR}=2.03)$, magnesium exposure $(\mathrm{aOR}=1.82)$, and postpartum hemorrhage ( $\mathrm{aOR}$ $=2.57$ ), whereas treated cases were associated with increased blood transfusion $(\mathrm{aOR}=4.81)$, severe perineal lacerations $(\mathrm{aOR}=2.93)$, and postpartum hemorrhage $(\mathrm{aOR}=3.85)$, but decreased risk of cesarean deliveries $(\mathrm{aOR}=0.59)$. Independent neonatal outcomes included small for gestational age $(\mathrm{aOR}=3.04)$, meconiumstained fluid ( $a O R=1.85 ; 2.61)$, respiratory failure $(a O R=5.84)$, neonatal adaptation syndrome $(\mathrm{aOR}=11 ; 10.2)$, and neonatal seizures $(\mathrm{aOR}=12.3)$ in treated cases, whereas untreated cases were associated with hypoxia $(\mathrm{aOR}=2.83)$, low Apgar score $(\mathrm{aOR}=3.82)$, and encephalopathy $(\mathrm{aOR}=18.3)$. Exposure to multiple psychotropic medications independently increased the risk of neonatal adaptation syndrome, neonatal length of stay, and hypoglycemia.

Conclusion Untreated cases were associated with increased maternal adverse outcomes, whereas treated cases were associated with more adverse neonatal outcomes when compared with unaffected pregnancies.
\end{abstract}

received

August 15, 2017

accepted after revision

February 23, 2018
DOI https://doi.org/

10.1055/s-0038-1661379.

ISSN 2157-6998.
Copyright $\odot 2018$ by Thieme Medical

Publishers, Inc., 333 Seventh Avenue,

New York, NY 10001, USA.

Tel: +1(212) 584-4662.
License terms

ㄷ) (i) $\ominus$ (\$) 
Identifying risk factors for mental disorders that may affect pregnancy is an important prerequisite in developing interventions to lower adverse maternal and neonatal outcomes. The estimated prevalence of perinatal anxiety and depression varies in published studies and is estimated to be between 7 and 20\%., ${ }^{1,2}$ Previous studies have reported associations of perinatal anxiety and depression with obstetrical and neonatal factors such as substance abuse, socioeconomic factors, and medical complications of pregnancy, small for gestational age (SGA), ${ }^{1-5}$ and postpartum depression. However, lacking from the literature are comprehensive reports from the same cohort simultaneously studying prenatal, intrapartum, and neonatal outcomes to assess associations of untreated and treated anxiety and depression in comparison to unaffected pregnancies.

An important consideration is the medical management of these mental disorders in pregnancy and the potential consequences. ${ }^{6}$ Hanley et al estimated that $10.3 \%$ of all pregnancies may have some exposure to psychotropic medications. ${ }^{6}$ Currently, psychotropic medications require more extensive risk-benefit analyses to determine efficacy and safety in pregnancy. For example, evidence from a large study of publicly insured pregnant women ${ }^{7}$ and a recent meta-analysis ${ }^{8}$ are consistent with a potential increased risk of persistent pulmonary hypertension associated with maternal use of selective serotonin reuptake inhibitors (SSRIs) in late pregnancy. However, the absolute risk was noted to be small, and the risk increase appears more modest than suggested in previous studies. Other studies have shown a significantly increased risk of spontaneous abortion, preterm birth, and low birth weight. All of the observed risks were of a very low magnitude, and the clinical significance of these results is unknown. ${ }^{9}$ However, benefits of antidepressant medication in pregnancy include prevention of possible relapse of illness, with resultant hospital admission and suicidal ideations. ${ }^{9}$ Consequently, studies analyzing the interactive effect of psychotropic treatment on mental disorders with obstetrical and neonatal outcomes are required for counseling and clinical management.

Therefore, the objectives of this study were (1) to determine the independent prenatal, intrapartum, and neonatal correlates of pregnancies affected by depression and anxiety and (2) to evaluate the differences in outcomes between cases of depression and anxiety treated and not treated with psychotropic medications when compared with unaffected pregnancies.

\section{Materials and Methods}

This was a retrospective cohort study of all women with singleton pregnancies and their newborns who delivered from January 1, 2013, through December 31, 2014, in the Beaumont Health System. An obstetrical outcomes database was developed using a combination of International Classification of Diseases, ninth edition (ICD-9), coding and direct querying of the electronic medical record. The extracted data were audited and verified by manual chart review as indi- cated. The database included a maximum of 18 months hospital follow-up data.

Beaumont Health is a not-for-profit health system located in suburban southeastern Michigan and services a diverse population of approximately 5 million, comprising Non-Hispanic Whites (68.5\%), Blacks (21.6\%), Asians (3.6\%), and Hispanics (3.9\%).

Cases of anxiety and depression were retrieved using the ICD-9 codes 293.84, 300.00, 300.01, 300.02, and 300.09 for anxiety and 296.2, 296.3, and 311 for depression (please refer to the -Appendix $\mathbf{A}$ for more details). A comprehensive manual chart review was completed on all women identified as having depression or anxiety to determine treatment with psychotropic medications during the antepartum period. This was accomplished by review of the admission history, and physical activity as well as medication prescription during the hospital admission. Those who had a positive history and were prescribed psychotropic medication during the hospital admission were classified as "treated," whereas those who were not prescribed medication during the hospital admission were classified as "untreated." Cases of treated and untreated anxiety, depression, and comorbid anxiety and depression were compared with unaffected pregnancies regarding various prenatal, intrapartum, and maternal and neonatal factors and outcomes that were selected based on literature reviews and biological plausibility.

Prenatal factors that were analyzed included maternal age, body mass index, gestational age, self-reported race or ethnicity, parity, marital status, medical insurance type, tobacco use, drug use, diabetes (gestational and pregestational), hypertensive disorders (chronic hypertension, gestational hypertension, severe preeclampsia, and superimposed preeclampsia), and previous cesarean delivery.

Intrapartum and maternal factors included induction of labor, exposure to cervical ripening agents (misoprostol, dinoprostone, oxytocin, and Foley catheter), abnormal fetal heart rate (ICD-9 code 659.71), systolic and diastolic blood pressures, pulse rate, temperature, exposure to intravenous antibiotics (gentamicin or clindamycin), exposure to intravenous antihypertensive medications (labetalol and hydralazine), exposure to magnesium, exposure to insulin, preterm birth less than 37 weeks' gestation, type of delivery, severe perineal laceration, chorioamnionitis, placental abruption, postpartum hemorrhage, blood transfusion, postpartum readmission, preterm rupture of membranes, shoulder dystocia, and length of stay (LOS).

Neonatal variables included infant sex, birth weight, Apgar scores, meconium-stained amniotic fluid, birth defects, admission to the neonatal intensive care unit (NICU), sepsis, persistent pulmonary hypertension, respiratory failure, hypoxia, hypoglycemia, seizure, encephalopathy, abstinence syndrome, umbilical arterial and venous blood gases, LOS, and a composite neonatal adverse outcome consisting of 5-minute Apgar score less than 4 , umbilical artery $\mathrm{pH}$ less than 7 , umbilical artery base excess greater than -12 , sepsis, seizures, encephalopathy, and respiratory failure. Please refer to the Appendix for a complete list of ICD-9 codes used. 
Univariate analyses of associations between maternal and neonatal factors and psychiatric diagnoses were performed by Student's $t$-test and chi-square test for continuous and categorical data, respectively. The associations that were significant on univariate analysis were further evaluated by multinomial logistic regression comparing each group to the unaffected referent pregnancies to calculate adjusted odds ratios (aORs) controlling for baseline differences. Because of the large numbers of cases and to assure clinical relevance, we only reported odds ratios greater than or equal to 1.5 or less than 0.9 . All statistical analyses were performed using SAS version 9.4 (SAS Institute, Cary, NC). This study was approved by our hospital's institutional review board.

\section{Results}

A total of 19,072 unique maternal-neonatal pairs were assessed for eligibility. Of these, 915 were excluded for multiple gestations and 2,665 for incomplete records, the majority of which were because of missing cord gas results. The remaining 15,492 (81.2\%) deliveries were analyzed for this study. Of these, depression or anxiety was present in 978 (6.3\%), including anxiety alone $(n=453 ; 2.9 \%)$, depression alone $(n=305 ; 2 \%)$, and comorbid depression and anxiety ( $n=220 ; 1.4 \%)$. These cases were further subdivided based on treatment with psychotropic medications as follows: treated anxiety alone $(n=101)$, treated depression alone $(n=123)$, treated comorbid depression and anxiety $(n=92)$, untreated anxiety alone $(n=351)$, untreated depression alone $(n=182)$, and untreated comorbid depression and anxiety $(n=129)$. These groups were compared with the 14,514 (93.7\%) unaffected reference pregnancies.

Considering significant maternal characteristics, patients with anxiety and depression were more likely to self-report as Non-Hispanic Whites, use tobacco and illegal substances, and be unmarried. Additionally, both treated depression and untreated depression were associated with public health insurance, multiparity, and hypertension. Both treated and untreated comorbid anxiety and depression were associated with hypertension, whereas treated comorbid anxiety and depression were associated with diabetes and untreated with public insurance. Only untreated anxiety was associated with decreased odds of previous cesarean delivery. Maternal age, body mass index, gestational age, and infant gender were similar across groups ( - Table $\mathbf{1}$ ).

Significant independently associated intrapartum and maternal factors are listed in -Table 2. This shows that untreated anxiety was associated with increased risks of cesarean deliveries $(\mathrm{aOR}=1.69)$, induction of labor $(\mathrm{aOR}=2.02)$, and longer maternal $\mathrm{LOS}(\mathrm{aOR}=1.85)$, risks not present among patients with treated anxiety. Those with untreated depression had higher odds of magnesium exposure $(\mathrm{aOR}=1.82)$ and readmission within 6 weeks of delivery $(\mathrm{aOR}=2.40)$, whereas treated depression was associated with decreased odds of cesarean delivery $(\mathrm{aOR}=0.59)$ but increased odds of requiring a blood transfusion $(\mathrm{aOR}=4.81)$. Untreated comorbid anxiety and depression were associated with increased risks of cesarean delivery ( $\mathrm{aOR}=1.59$ ), fever $>38^{\circ} \mathrm{C}(\mathrm{aOR}=2.03)$, postpartum hemorrhage ( $\left.\mathrm{aOR}=2.57\right)$, and maternal LOS $(\mathrm{aOR}=1.96)$, whereas treated comorbid anxiety and depression were associated with increased risks of severe perineal lacerations $(\mathrm{aOR}=2.93)$ and postpartum hemorrhage ( $\mathrm{aOR}=3.85$ ). There were no significant associations with abnormal fetal heart rate ICD-9 code, systolic or diastolic blood pressure, pulse rate, exposure to intravenous antibiotics, exposure to intravenous antihypertensive medications, insulin therapy, preterm birth before 37 weeks' gestation, operative vaginal delivery, chorioamnionitis, placental abruption, preterm rupture of membranes, and shoulder dystocia.

Significant independent associations of neonatal outcomes are listed in $\boldsymbol{-}$ Table 3 . This showed that untreated anxiety was associated with 5-minute Apgar score $<4 \quad(\mathrm{aOR}=3.82)$ whereas treated anxiety was associated with meconiumstained amniotic fluid $(\mathrm{aOR}=1.85)$, respiratory failure $(\mathrm{aOR}=5.84)$, and neonatal abstinence syndrome $(\mathrm{aOR}=11)$. Neonates of mothers with untreated depression had increased odds of hypoxia ( $\mathrm{aOR}=2.83$ ), but treated depression was associated with neonatal seizures $(\mathrm{aOR}=12.3)$ and neonatal abstinence syndrome $(\mathrm{aOR}=10.2)$. Untreated comorbid anxiety and depression were associated with encephalopathy $(\mathrm{aOR}=15)$, whereas treated comorbid disease was associated with increased odds of being SGA $(\mathrm{aOR}=3.04)$, meconiumstained amniotic fluid $(\mathrm{aOR}=2.61)$, arterial base excess greater than $-12(\mathrm{aOR}=3.42)$, and venous base excess greater than $-12(\mathrm{aOR}=5.62)$. There were no significant differences in admission to the NICU, birth defects, persistent pulmonary hypertension, sepsis, hypoglycemia, arterial and venous $\mathrm{pH}$ $<7$, LOS, and the neonatal composite adverse outcome.

Psychotropic medications were used by 339 subjects (34.6\%). The most common medications were SSRIs ( $n=271 ; 79.9 \%)$, benzodiazepines $(n=54 ; 15.9 \%)$, and other antidepressants $(n=35 ; 10.3 \%)$. Other medications included serotonin-norepinephrine reuptake inhibitors ( $n=18 ; 5.7 \%)$, antiepileptics ( $n=13 ; 4.4 \%)$, and antipsychotics $(n=10 ; 3.2 \%)$. A single drug was used by $285(84.1 \%)$ and multiple drugs by 54 (15.9\%). We did a subanalysis and compared those with multiple drugs (polypharmacy) with those who used single psychotropic drugs regarding neonatal outcomes. On univariate analysis, neonates born of mothers with multiple prenatal psychotropic drug use were significantly more likely to be SGA and have neonatal hypoglycemia, neonatal abstinence syndrome, NICU admission, and longer neonatal LOS; however, on adjusted regression analysis, NICU admission and neonatal LOS were no longer significant ( - Table 4 )

\section{Discussion}

In this hospital system, the prevalence of anxiety and depression overall was $6.3 \%$, which is somewhat lower than observed in other studies. ${ }^{6}$ This is probably because most previous reports used self-reported screening instruments in the identification of cases-a process that may overestimate with a resultant higher prevalence in comparison to this study, which used ICD-9 coding and detailed chart review for identification of cases. ${ }^{1,10}$ However, the low 


\begin{tabular}{|c|c|c|c|c|c|c|c|c|c|c|c|c|c|}
\hline 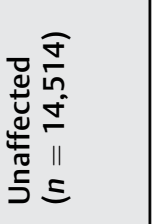 & $\begin{array}{l}\sim \\
\dot{n} \\
+ \\
\bar{m}\end{array}$ & $\begin{array}{l}-\dot{b} \\
+1 \\
\stackrel{n}{+} \\
\dot{m}\end{array}$ & $\begin{array}{c}r \\
\dot{r} \\
+ \\
\bar{\sigma} \\
\dot{m}\end{array}$ & $\begin{array}{l}\frac{\widehat{\sigma}}{\omega} \\
\underline{\omega} \\
\stackrel{N}{a} \\
\sigma\end{array}$ & 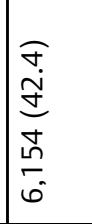 & 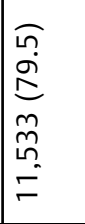 & 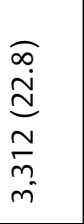 & $\begin{array}{l}\frac{\Omega}{10} \\
\dot{f} \\
\text { f }\end{array}$ & 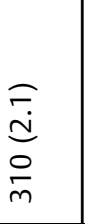 & 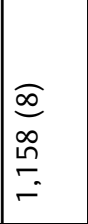 & 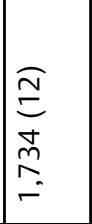 & $\begin{array}{l}\widehat{\bar{d}} \\
m \\
\stackrel{m}{0} \\
m\end{array}$ & 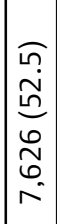 \\
\hline 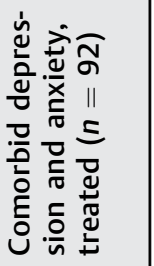 & $\begin{array}{l}n \\
\dot{n} \\
+ \\
m \\
\tilde{m} \\
\tilde{m}\end{array}$ & $\begin{array}{l}\sigma \\
\dot{b} \\
+1 \\
\overline{\dot{n}} \\
\tilde{m}\end{array}$ & 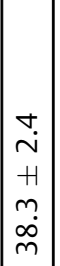 & 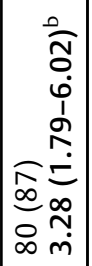 & 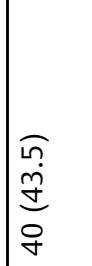 & 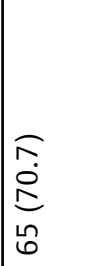 & $\begin{array}{l}\text { f. } \\
\dot{0} \\
\stackrel{0}{0} \\
\stackrel{\infty}{N}\end{array}$ & 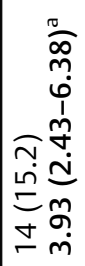 & 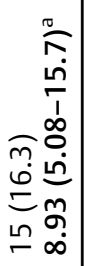 & 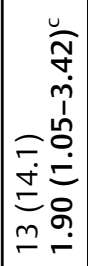 & 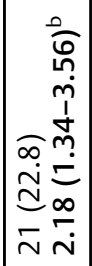 & 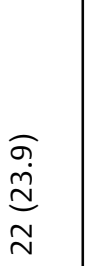 & $\begin{array}{c}\sigma \\
\infty \\
\infty \\
\dot{+} \\
\qquad \mathfrak{\gamma}\end{array}$ \\
\hline 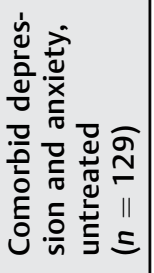 & 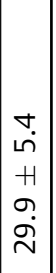 & $\begin{array}{l}r \\
\dot{r} \\
H \\
r \\
m \\
m\end{array}$ & $\begin{array}{l}\hat{i} \\
i \\
+ \\
\infty \\
\infty \\
\dot{m} \\
\end{array}$ & 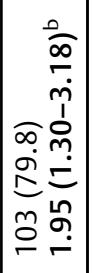 & 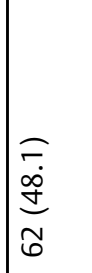 & 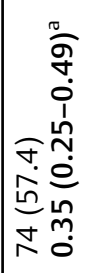 & 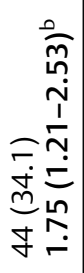 & 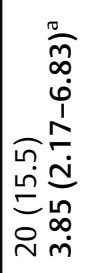 & 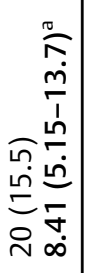 & 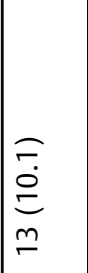 & 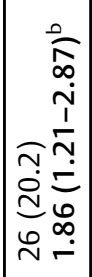 & 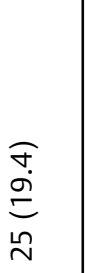 & 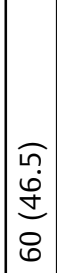 \\
\hline 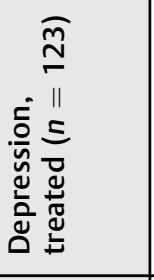 & $\begin{array}{l}\bar{b} \\
+1 \\
\llcorner \\
\bar{\Gamma}\end{array}$ & $\begin{array}{l}m \\
i n \\
+ \\
n \\
i \\
m\end{array}$ & $\begin{array}{c}\sim \\
i \\
+ \\
m \\
m \\
\infty \\
m \\
\end{array}$ & 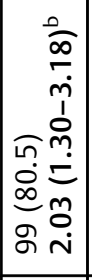 & 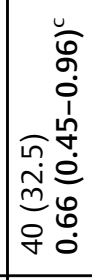 & 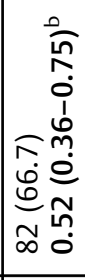 & 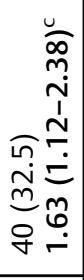 & 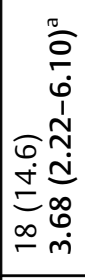 & $\underset{\text { in }}{\underset{\dot{J}}{(j)}}$ & $\begin{array}{l}\bar{n} \\
\stackrel{n}{a} \\
\sigma\end{array}$ & 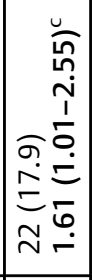 & $\begin{array}{l}\underset{\infty}{\infty} \\
\underset{d}{d} \\
\stackrel{\infty}{N}\end{array}$ & $\begin{array}{l}\overparen{\sigma} \\
\dot{0} \\
\stackrel{0}{0} \\
\tilde{\sigma}\end{array}$ \\
\hline 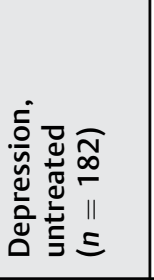 & $\begin{array}{l}\infty \\
\dot{1} \\
+ \\
\hat{n} \\
\stackrel{n}{N}\end{array}$ & $\begin{array}{l}\infty \\
\dot{\omega} \\
H \\
\sim \\
\\
m\end{array}$ & $\left|\begin{array}{c}r \\
i \\
H \\
r \\
\hat{\infty} \\
m\end{array}\right|$ & 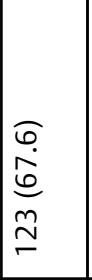 & 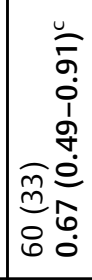 & 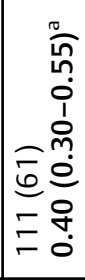 & 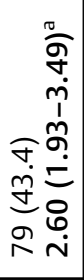 & 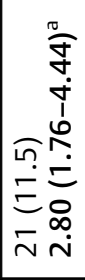 & 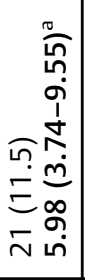 & $\begin{array}{c} \\
\widehat{m} \\
a \\
\tilde{a} \\
\end{array}$ & 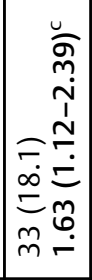 & $\begin{array}{l}\underset{\sigma}{\tilde{d}} \\
\underset{\mathfrak{y}}{m}\end{array}$ & $\begin{array}{l}\sigma \\
\hat{n} \\
\stackrel{n}{n} \\
\infty \\
\sigma\end{array}$ \\
\hline 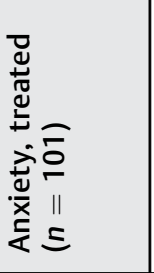 & \begin{tabular}{|l|} 
in \\
+ \\
+ \\
$\dot{j}$ \\
$\bar{m}$ \\
\end{tabular} & $\begin{array}{l}\infty \\
\dot{0} \\
H \\
\tilde{m}\end{array}$ & $\mid \begin{array}{l}0 \\
\sim \\
+ \\
+ \\
0 \\
\infty \\
m\end{array}$ & 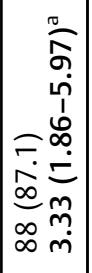 & 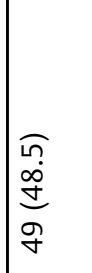 & 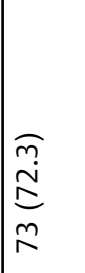 & $\begin{array}{l}\underset{\infty}{\infty} \\
\underset{d}{N} \\
\end{array}$ & 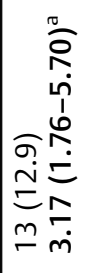 & 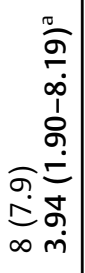 & $\underset{\substack{a\\
}}{\stackrel{a}{=}}$ & 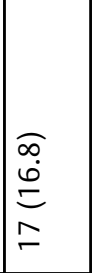 & 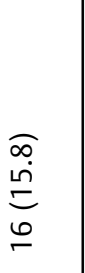 & 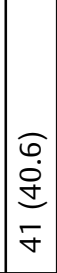 \\
\hline 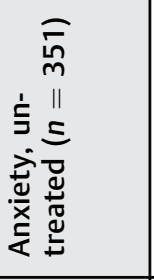 & 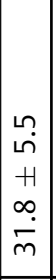 & $\begin{array}{l}\sim \\
\dot{b} \\
+1 \\
\underline{\dot{ }} \\
\dot{m}\end{array}$ & $\begin{array}{l}\sim \\
\sim \\
\sim \\
+1 \\
\sigma \\
m\end{array}$ & 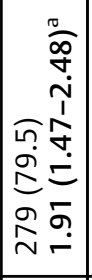 & 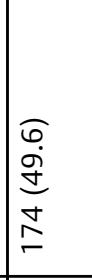 & 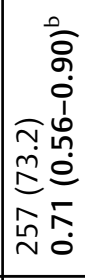 & 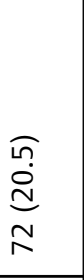 & 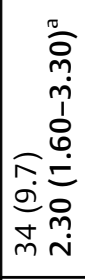 & 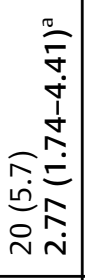 & 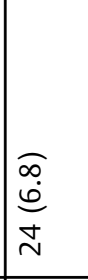 & 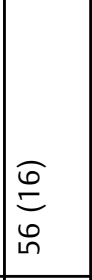 & 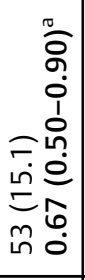 & 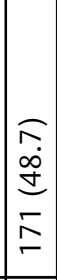 \\
\hline $\begin{array}{l}\frac{0}{d 0} \\
\frac{\pi}{\frac{\pi}{n}} \\
\frac{5}{>}\end{array}$ & 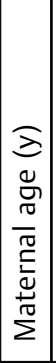 & 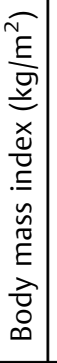 & 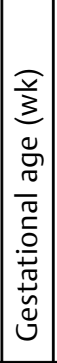 & 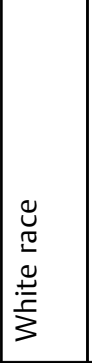 & 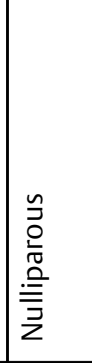 & 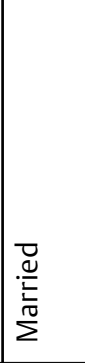 & 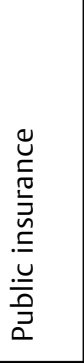 & $\begin{array}{l}\tilde{y} \\
\check{I} \\
\stackrel{u}{u} \\
\tilde{0} \\
\stackrel{0}{\circ}\end{array}$ & 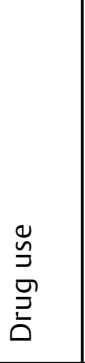 & $\begin{array}{l}\tilde{y} \\
\stackrel{\Delta}{0} \\
\frac{\pi}{0} \\
\stackrel{0}{0}\end{array}$ & 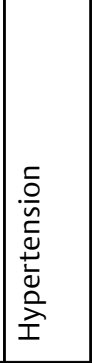 & 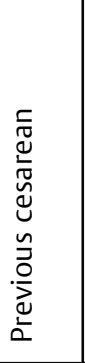 & 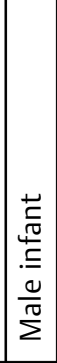 \\
\hline
\end{tabular}




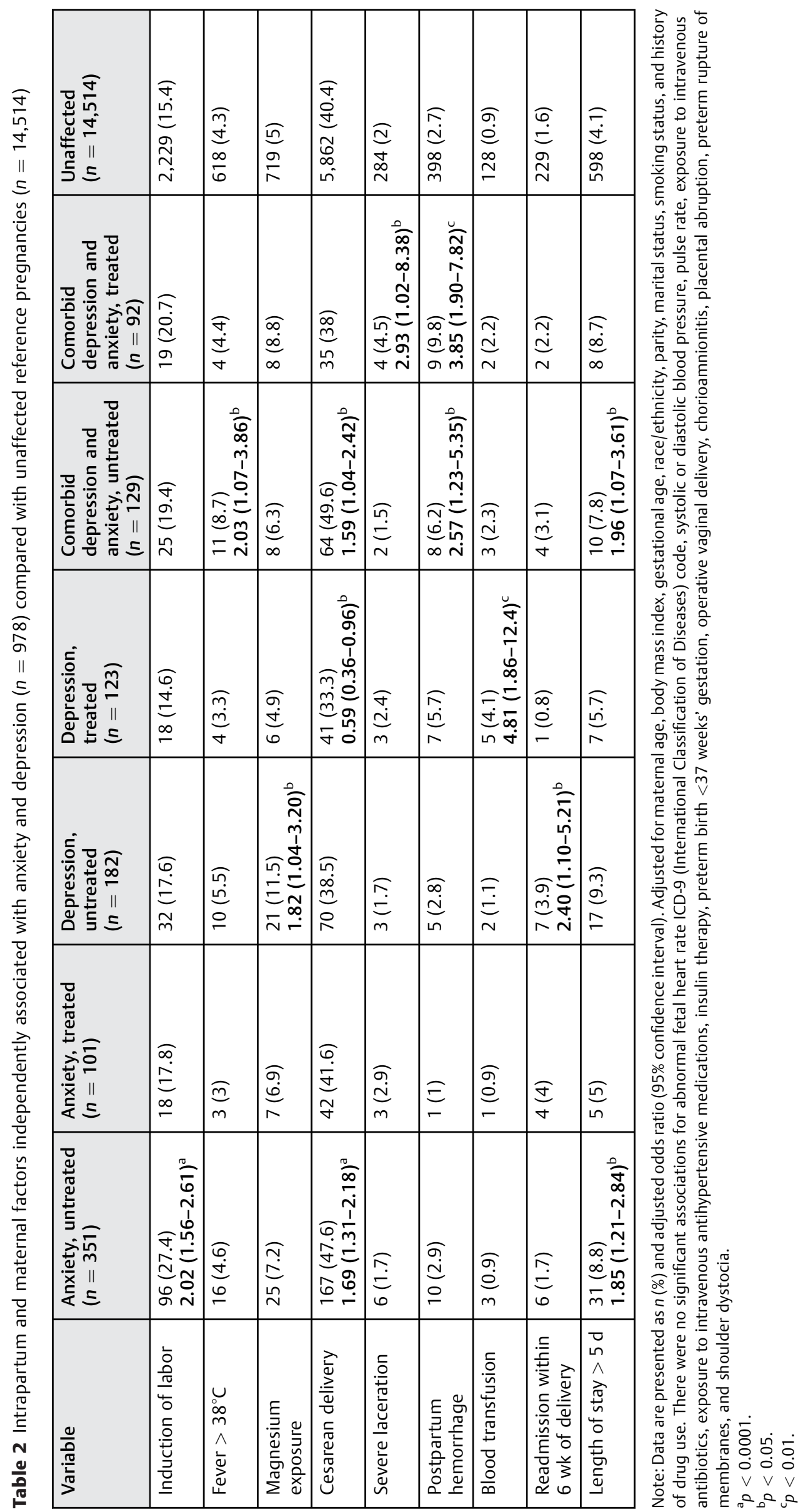




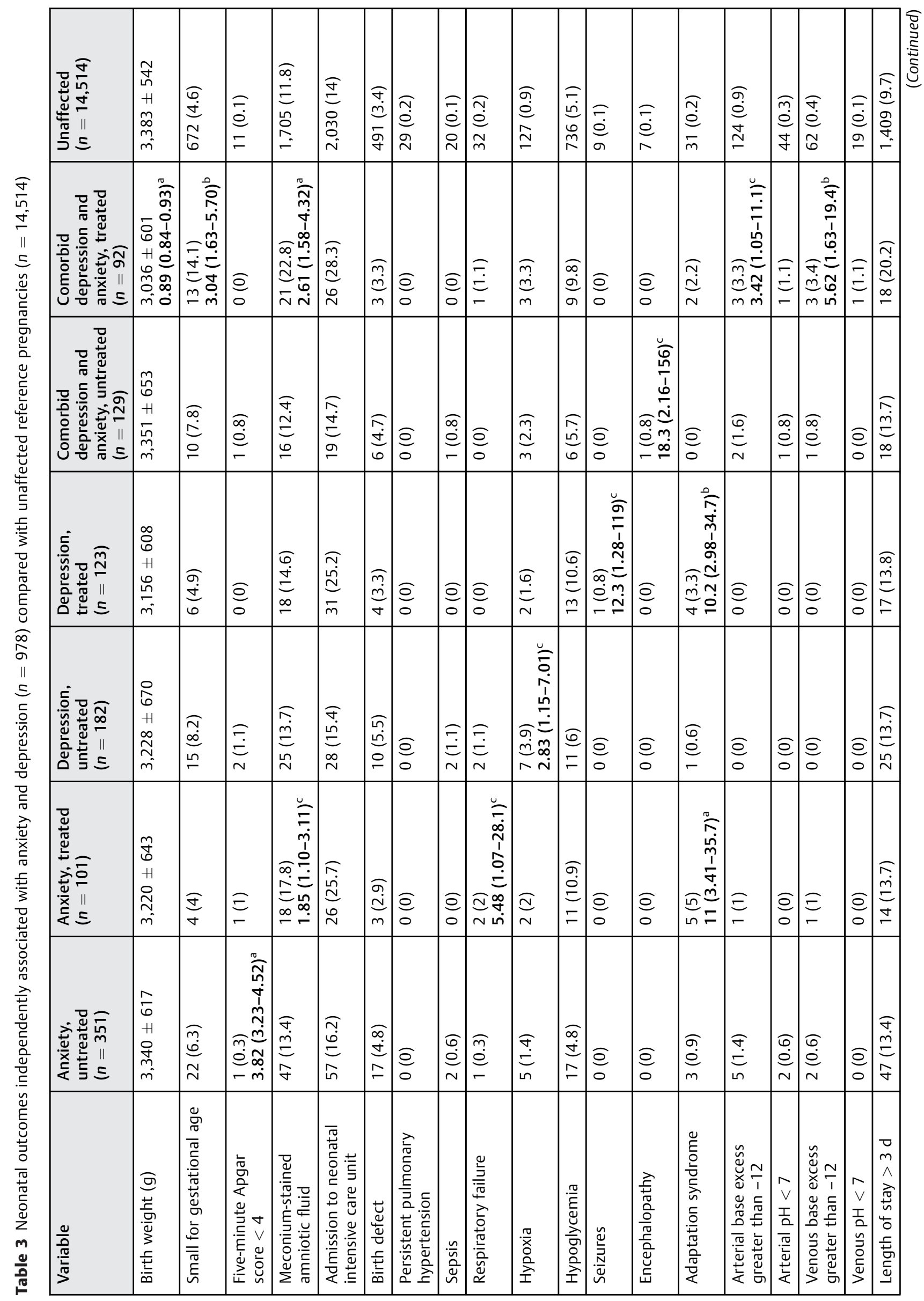



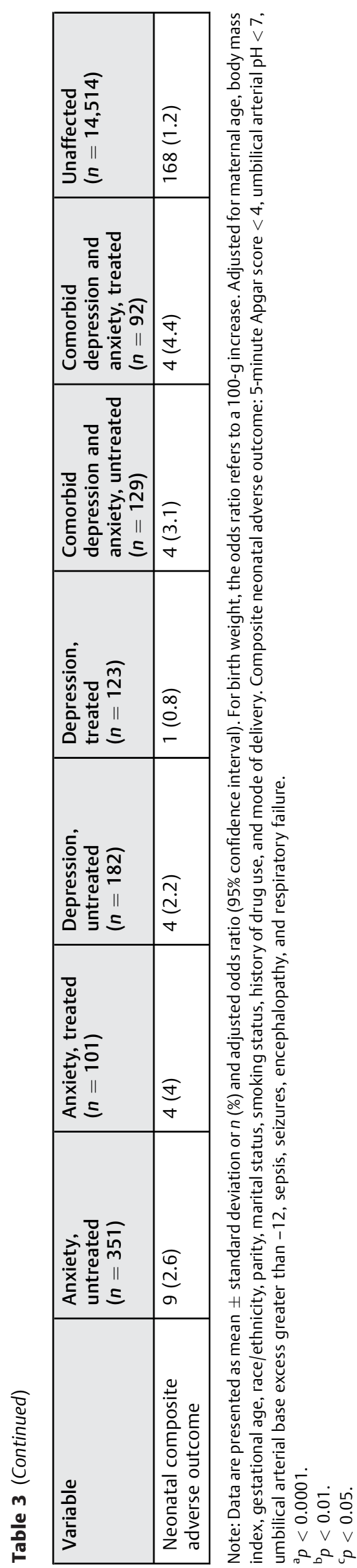

prevalence noted in this study could also be because of potential misclassification of both "noncases" and "cases." It is likely that there are several missed cases of depression or anxiety in the reference group. The findings in this study support other previous reports showing associations between anxiety/depression and smoking, substance abuse, and unmarried status. ${ }^{11-17}$ But in contrast to most other studies that have likewise shown associations with minority ethnic groups, our study showed an association with NonHispanic white women. ${ }^{11-17}$ The increased risk of perinatal anxiety and depression in minority groups has been explained by stress-related discrimination in addition to financial and social disadvantages. ${ }^{18}$ However, another report in support of our findings demonstrated higher levels of depression among White mothers compared with black and Hispanic mothers as well as women of other ethnicities. ${ }^{19}$ As an explanation, other studies have demonstrated that prolonged and repeated exposure to adversity might provide minority women with greater resilience and skills to manage psychological distress in addition to having higher levels of social support, spirituality, and self-esteem resources. ${ }^{20}$ Correspondingly, our study highlights that Non-Hispanic Whites may also be at increased risk of anxiety and depression during pregnancy and the need for universal screening of all pregnant women.

In this study, untreated prenatal anxiety and depression were associated with more adverse maternal outcomes than treated ( 7 vs. 3, respectively) when both groups were compared with those without anxiety and depression. Both prenatal anxiety and comorbid anxiety and depression when untreated were associated with an increased risk of cesarean birth, whereas treated depression demonstrated a decreased risk. A past history of cesarean delivery has been found to be associated with a high incidence of antenatal anxiety and depression. ${ }^{21,22}$ However, our study did show a decreased risk of previous cesarean delivery with those with untreated anxiety. The association of anxiety with previous cesarean birth may not be very reliable since its validity would depend on the following: (1) if the patient did have a diagnosis of anxiety during the previous pregnancy and (2) if she was treated in that pregnancy. Our study's finding of increased risk of cesarean birth with untreated prenatal anxiety and depression with a corresponding decrease when treated is intriguing. Other studies have found no association between mode of delivery and antenatal depression, ${ }^{23,24}$ whereas another previous study did show an association between untreated depression and cesarean section..$^{25} \mathrm{~A}$ recent systemic review showed that those requesting elective cesarean sections had higher antepartum depression and anxiety levels but no different postpartum depression levels than women who delivered vaginally. ${ }^{26}$ It is important to note that in a Finnish study on major depression in pregnancy, after a history of depression, the second strongest associated factor for major depression was fear of childbirth, with a 2.6 -fold increased prevalence. ${ }^{10}$ Furthermore, other studies have shown that patients with antenatal anxiety and depression have a fear of childbirth and show a preference for an elective cesarean delivery. ${ }^{1}$ These findings 
Table 4 Significant associations of prenatal single versus multiple psychotropic drug use with neonatal outcomes $(n=339)$

\begin{tabular}{|l|l|l|l|l|}
\hline Neonatal factor & $\begin{array}{l}\text { Single psychotropic } \\
\text { drug, } \boldsymbol{n}=\mathbf{2 8 5}\end{array}$ & $\begin{array}{l}\text { Multiple psychotropic } \\
\text { drugs, } \boldsymbol{n}=\mathbf{5 4}\end{array}$ & $\begin{array}{l}\text { Univariate } \boldsymbol{p} \text {-value } \\
\text { (OR) }\end{array}$ & $\begin{array}{l}\text { Independent } \boldsymbol{p} \text {-value } \\
\text { (OR) [95\%Cl] }\end{array}$ \\
\hline Male gender & $134(47 \%)$ & $29(53.7 \%)$ & $\mathrm{ns}$ & $\mathrm{ns}$ \\
\hline Birth weight & $3,058.86(43.6)$ & $2,877.17(100.58)$ & $\mathrm{ns}$ & $\mathrm{ns}$ \\
\hline $\begin{array}{l}\text { Neonatal } \\
\text { hypoglycemia }\end{array}$ & $25(8.8 \%)$ & $11(20.4 \%)$ & $(2.66)^{\mathrm{a}}$ & $(2.6)[1.04-6.55]^{\mathrm{a}}$ \\
\hline $\begin{array}{l}\text { Neonatal abstinence } \\
\text { syndrome }\end{array}$ & $3(1.1 \%)$ & $8(14.8 \%)$ & $(16.348)^{\mathrm{b}}$ & $(8.3)[1.72-39.9]^{\mathrm{c}}$ \\
\hline $\begin{array}{l}\text { Admission to neonatal } \\
\text { intensive care unit }\end{array}$ & $80(28.1 \%)$ & $25(46.3 \%)$ & $(2.2)^{\mathrm{a}}$ & $\mathrm{ns}$ \\
\hline SGA & $18(6.3 \%)$ & $8(14.8 \%)$ & $(2.57)^{\mathrm{a}}$ & \\
\hline Length of stay > 3d & $50(17.5 \%)$ & $22(40.7 \%)$ & $(3.23)^{\mathrm{b}}$ & $(3.2)[1.15-9.09]^{\mathrm{a}}$ \\
\hline
\end{tabular}

Abbreviations: $\mathrm{Cl}$, confidence interval; ns, not significant; OR, odds ratio; SGA, small for gestational age.

Note: There were no significant associations with the neonatal composite score, arterial $\mathrm{pH}<7$, venous base excess greater than -12 , venous $\mathrm{pH}<7$, 5-minute Apgar score $<4$, and meconium-stained amniotic fluid. The independent $p$-value was adjusted for maternal age, body mass index, gestational age, race/ethnicity, parity, marital status, smoking status, history of drug use, and mode of delivery.

${ }^{a} p<0.05$.

${ }^{\mathrm{b}} \mathrm{p}<0.0001$.

${ }^{c} p<0.01$.

suggest a need for greater attention to continuous assessment of psychological well-being among women, especially those requesting elective cesarean birth, as a potential intervention for the current major public health concern with the increasing prevalence of cesarean delivery. Further prospective studies are required to test this hypothesis.

In this study, treated prenatal anxiety and depression were also associated with more adverse neonatal outcomes than untreated cases ( 7 versus 3 respectively) when both groups were compared with those without anxiety and depression. The increased association of treatment of anxiety/depression with psychotropic drugs during pregnancy with neonatal adaptation syndrome supports previous findings. ${ }^{9}$ Furthermore, treatment of comorbid anxiety and depression was associated with a threefold risk for SGA neonate, whereas there was no association with preterm birth. The impact of antidepressants on fetal growth has been evaluated with inconclusive results, with most studies reviewed lacking adequate control groups with heterogeneity of outcomes. ${ }^{9}$ In contrast to our findings, a recent study demonstrated an association of a positive screen for depression using Edinburgh Postnatal Depression Scale (EPDS) with preterm birth and SGA, but it noted that such risk was not apparent among women who were treated with an antidepressant medication. ${ }^{27}$ The difference in results may be partially explained by methodology since in our study we identified patients with depression or anxiety based on ICD9 coding and we compared identified patients with depression or anxiety with those who were not unaffected based on the use of psychotropic drugs. In the aforementioned study, patients were identified by EPDS, and all patients who received antidepressants (either screen-positive or screennegative) were compared with all those who did not use antidepressants (both screen-positive and screen-negative).
Meconium-stained fluid, which is associated with intrauterine hypoxia, was also increased in treated cases in our study. Our findings and those of previous reports do support that psychotropic medications in pregnancy are associated with some neonatal morbidity.

It should also be noted that untreated anxiety and depression were not without neonatal morbidity and were associated with increased risks including low Apgar scores and neonatal hypoxia. It conceivable that the increased maternal morbidity associated with untreated anxiety and depression noted in this study and previous works can predispose to neonatal morbidity. ${ }^{1,10,27,28}$ For example, maternal fever, which is a predictor of neonatal sepsis and morbidity, was only associated with untreated comorbid anxiety and depression. Furthermore, studies in pregnant women have shown increased relapse rates in those who discontinued their psychotropic medication during pregnancy, with increased risks of hospitalization and suicide ideation. ${ }^{29,30}$ Neonatal adaptation syndrome, which is the main neonatal morbidity associated with antidepressant use, is known to be transient with the symptoms resolving within a week with no apparent long-term adverse effects. ${ }^{9}$ Correspondingly, the risks associated with pharmacological treatment must be balanced with the effects of untreated antenatal maternal depression on the mother-fetus dyad, which have the potential to be devastating.

\section{Limitations}

There are several limitations to this study. This is a retrospective cohort study design, which has inherent limitations including missing data and accuracy of data. Specifically, the used of ICD-9 codes for identifying cases has intrinsic limitations, namely, accuracy of diagnostic code, potentially 
nonactive diagnoses, and noncoded cases (i.e., unidentified cases). The latter is supported by the fact that the prevalence of anxiety/depression in our study (6.3\%) is lower than that reported in the literature. ${ }^{31}$ Additionally, there were no data regarding the adequacy of prenatal care, duration of anxiety and depression, and the level of control entering the pregnancy. However, we did manual review and audits of the charts for verification and confirmation of the data. We also performed multivariate logistic regression to eliminate potential confounders, but it is possible that there are other factors that were not measured. Such potential cofounders include quantity, timing, and duration of psychotropic medication therapy. We also did not assess postpartum depression; however, multiple studies have already confirmed the strong association of prenatal anxiety and depression with postpartum depression. ${ }^{30}$ We also did not directly compare treated cases versus untreated cases of anxiety and depression, which would have provided differential effects of the exposure of medications, but compared against unaffected pregnancies to determine the baseline burden of disease and treatment. Moreover, there are always concerns with medication compliance, and modest associations regarding medication treatment and outcomes can be attributed to misclassification because nondifferential misclassification of the exposure or the outcome will tend to bias results toward the null hypothesis. ${ }^{32,33}$ Another limitation is that many of the neonatal adverse outcomes such as respiratory failure, neonatal seizures, encephalopathy, low Apgar score, and abnormal blood gases had very small numbers with correspondingly very wide $95 \%$ confidence intervals. Hence, these findings, though significant, may not be clinically relevant. Furthermore, this is a single-system study in southeastern Michigan; therefore, the findings may not be applicable to different centers with different populations. Strengths of this study include assessment of a large cohort of mother-fetus dyads using uniform methodology and ascertainment of depression, anxiety, and maternal and neonatal outcomes by the electronic health record with detailed review, thus avoiding the risk of recall bias and overestimation seen in some earlier studies. Through detailed categorization of these pregnant women, we were able to control for important confounding variables associated with maternal and neonatal morbidity.

\section{Conclusion}

In contrast to the majority of previous studies, Non-Hispanic White mothers were at a greater risk of prenatal anxiety and depression in addition to other social disadvantaged factors, substance use, and medical disorders. Women with untreated anxiety and depression in pregnancy had more adverse maternal outcomes. Untreated anxiety appeared to be a risk factor for induction of labor and cesarean delivery. Treated patients with anxiety and depression had more neonatal adverse outcomes; however, untreated patients still had some neonatal morbidity presumably as a consequence of significant maternal morbidity. A third of women used psychotropic medications during pregnancy, with an increased risk of neonatal adaptation syndrome, which is known to be transient without long-time sequelae. The universal identification of women at risk of anxiety and depression during pregnancy may provide opportunities for therapy and promote well-being of mothers and babies.

\section{Presentation}

Paper was presented at the 2017 Annual Clinical and Scientific Meeting of the American College of Obstetrician and Gynecologists, San Diego, California.

\section{References}

1 Biaggi A, Conroy S, Pawlby S, Pariante CM. Identifying the women at risk of antenatal anxiety and depression: a systematic review. J Affect Disord 2016;191:62-77

2 Gavin NI, Gaynes BN, Lohr KN, Meltzer-Brody S, Gartlehner G, Swinson T. Perinatal depression: a systematic review of prevalence and incidence. Obstet Gynecol 2005;106(5 Pt 1):1071-1083

3 Fisher J, Cabral de Mello M, Patel V, et al. Prevalence and determinants of common perinatal mental disorders in women in low- and lower-middle-income countries: a systematic review. Bull World Health Organ 2012;90(02):139G-149G

4 Betts KS, Williams GM, Najman JM, Scott J, Alati R. The association between lower birth weight and comorbid generalised anxiety and major depressive disorder. J Affect Disord 2013;146(02):231-237

5 Howard LM, Molyneaux E, Dennis CL, Rochat T, Stein A, Milgrom J. Non-psychotic mental disorders in the perinatal period. Lancet 2014;384(9956):1775-1788

6 Hanley GE, Mintzes B. Patterns of psychotropic medicine use in pregnancy in the United States from 2006 to 2011 among women with private insurance. BMC Pregnancy Childbirth 2014;14:242

7 Huybrechts KF, Bateman BT, Palmsten K, et al. Antidepressant use late in pregnancy and risk of persistent pulmonary hypertension of the newborn. JAMA 2015;313(21):2142-2151

8 Grigoriadis S, Vonderporten EH, Mamisashvili L, et al. Prenatal exposure to antidepressants and persistent pulmonary hypertension of the newborn: systematic review and meta-analysis. BMJ 2014;348:f6932

9 Lorenzo L, Einarson A. Antidepressant use in pregnancy: an evaluation of adverse outcomes excluding malformation. Isr J Psychiatry Relat Sci 2014;51(02):94-104

10 Räisänen S, Lehto SM, Nielsen HS, Gissler M, Kramer MR, Heinonen S. Risk factors for and perinatal outcomes of major depression during pregnancy: a population-based analysis during 20022010 in Finland. BMJ Open 2014;4(11):e004883

11 Faisal-Cury A, Rossi Menezes P. Prevalence of anxiety and depression during pregnancy in a private setting sample. Arch Women Ment Health 2007;10(01):25-32

12 Fellenzer JL, Cibula DA. Intendedness of pregnancy and other predictive factors for symptoms of prenatal depression in a population-based study. Matern Child Health J 2014;18(10):2426-2436

13 Gavin AR, Melville JL, Rue T, Guo Y, Dina KT, Katon WJ. Racial differences in the prevalence of antenatal depression. Gen Hosp Psychiatry 2011;33(02):87-93

14 Melville JL, Gavin A, Guo Y, Fan MY, Katon WJ. Depressive disorders during pregnancy: prevalence and risk factors in a large urban sample. Obstet Gynecol 2010;116(05):1064-1070

15 Redshaw M, Henderson J. From antenatal to postnatal depression: associated factors and mitigating influences. J Womens Health (Larchmt) 2013;22(06):518-525

16 Shakeel N, Eberhard-Gran M, Sletner L, et al. A prospective cohort study of depression in pregnancy, prevalence and risk factors in a multi-ethnic population. BMC Pregnancy Childbirth 2015;15:5

17 Verreault N, Da Costa D, Marchand A, Ireland K, Dritsa M, Khalifé S. Rates and risk factors associated with depressive symptoms 
during pregnancy and with postpartum onset. J Psychosom Obstet Gynaecol 2014;35(03):84-91

18 Shen JJ, Lin F, Jackson T. Risk of prenatal depression: differences by race. Ethn Dis 2010;20(01):35-39

19 Prady SL, Pickett KE, Croudace T, et al. Psychological distress during pregnancy in a multi-ethnic community: findings from the born in Bradford cohort study. PLoS One 2013;8(04): e60693

20 Jesse DE, Walcott-McQuigg J, Mariella A, Swanson MS. Risks and protective factors associated with symptoms of depression in low-income African American and Caucasian women during pregnancy. J Midwifery Womens Health 2005;50(05):405-410

21 Kuo SY, Chen SR, Tzeng YL. Depression and anxiety trajectories among women who undergo an elective cesarean section. PLoS One 2014;9(01):e86653

22 Waqas A, Raza N, Lodhi HW, Muhammad Z, Jamal M, Rehman A. Psychosocial factors of antenatal anxiety and depression in Pakistan: is social support a mediator? PLoS One 2015;10(01):e0116510

23 Adewuya AO, Ola BA, Aloba OO, Dada AO, Fasoto OO. Prevalence and correlates of depression in late pregnancy among Nigerian women. Depress Anxiety 2007;24(01):15-21

24 Ajinkya S, Jadhav PR, Srivastava NN. Depression during pregnancy: prevalence and obstetric risk factors among pregnant women attending a tertiary care hospital in Navi Mumbai. Ind Psychiatry J 2013;22(01):37-40

25 Chung TK, Lau TK, Yip AS, Chiu HF, Lee DT. Antepartum depressive symptomatology is associated with adverse obstetric and neonatal outcomes. Psychosom Med 2001;63(05):830-834
26 Olieman RM, Siemonsma F, Bartens MA, Garthus-Niegel S, Scheele F, Honig A. The effect of an elective cesarean section on maternal request on peripartum anxiety and depression in women with childbirth fear: a systematic review. BMC Pregnancy Childbirth 2017;17(01):195

27 Venkatesh KK, Riley L, Castro VM, Perlis RH, Kaimal AJ. Association of antenatal depression symptoms and antidepressant treatment with preterm birth. Obstet Gynecol 2016;127(05):926-933

28 Einarson A, Selby P, Koren G. Abrupt discontinuation of psychotropic drugs during pregnancy: fear of teratogenic risk and impact of counselling. J Psychiatry Neurosci 2001;26(01):44-48

29 Cohen LS, Altshuler LL, Harlow BL, et al. Relapse of major depression during pregnancy in women who maintain or discontinue antidepressant treatment. JAMA 2006;295(05):499-507

30 Silverman ME, Reichenberg A, Savitz DA, et al. The risk factors for postpartum depression: a population-based study. Depress Anxiety 2017;34(02):178-187

31 Eke AC, Saccone G, Berghella V. Selective serotonin reuptake inhibitor (SSRI) use during pregnancy and risk of preterm birth: a systematic review and meta-analysis. BJOG 2016;123(12):1900-1907

32 Correa-Villaseñor A, Stewart WF, Franco-Marina F, Seacat H. Bias from nondifferential misclassification in case-control studies with three exposure levels. Epidemiology 1995;6(03):276-281

33 Keyloun KR, Hansen RN, Hepp Z, Gillard P, Thase ME, Devine EB. Adherence and persistence across antidepressant therapeutic classes: a retrospective claims analysis among insured US patients with major depressive disorder (MDD). CNS Drugs 2017;31(05): 421-432 
e156 Contribution of Untreated and Treated Anxiety/Depression Ogunyemi et al.

Appendix A ICD-9 codes

\begin{tabular}{|c|c|c|}
\hline Diagnosis & Code & Description \\
\hline \multicolumn{3}{|l|}{ Maternal } \\
\hline Abnormal fetal heart rate & 659.71 & Abnormality in fetal heart rate or rhythm, delivered \\
\hline \multirow[t]{5}{*}{ Anxiety } & 293.84 & Anxiety disorder in conditions classified elsewhere \\
\hline & 300.00 & Anxiety state, unspecified \\
\hline & 300.01 & Panic disorder without agoraphobia \\
\hline & 300.02 & Generalized anxiety disorder \\
\hline & 300.09 & Other anxiety states \\
\hline Chorioamnionitis & $658.4 x$ & Infection of amniotic cavity \\
\hline \multirow[t]{3}{*}{ Depression } & $296.2 x$ & Major depressive disorder, single episode \\
\hline & $296.3 x$ & Major depressive disorder, recurrent episode \\
\hline & 311 & Depressive disorder, not otherwise classified \\
\hline Diabetes, gestational & $648.8 x$ & Abnormal maternal glucose tolerance \\
\hline \multirow[t]{2}{*}{ Diabetes, pregestational } & $250 . x$ & Diabetes mellitus \\
\hline & $648.0 x$ & Diabetes mellitus of the mother complicating pregnancy \\
\hline \multirow[t]{8}{*}{ Hypertension, chronic } & 401.x & Essential hypertension \\
\hline & 402.x & Hypertensive heart disease \\
\hline & 403.x & Hypertensive chronic kidney disease \\
\hline & 404.x & Hypertensive heart and chronic kidney disease \\
\hline & 405.x & Secondary hypertension \\
\hline & $642.0 x$ & Benign essential hypertension complicating pregnancy \\
\hline & $642.1 x$ & $\begin{array}{l}\text { Hypertension secondary to renal disease complicating } \\
\text { pregnancy }\end{array}$ \\
\hline & $642.2 x$ & Other preexisting hypertension complicating pregnancy \\
\hline \multirow[t]{6}{*}{ Hypertension, gestational } & $642.3 x$ & Transient hypertension of pregnancy \\
\hline & $642.4 x$ & Mild or unspecified preeclampsia \\
\hline & $642.5 x$ & Severe preeclampsia \\
\hline & $642.6 x$ & Eclampsia \\
\hline & $642.7 x$ & $\begin{array}{l}\text { Preeclampsia or eclampsia superimposed on preexisting } \\
\text { hypertension }\end{array}$ \\
\hline & $642.9 x$ & Unspecified hypertension complicating pregnancy \\
\hline Placental abruption & $641.2 x$ & Premature separation of the placenta \\
\hline \multirow[t]{4}{*}{ Postpartum hemorrhage } & $666.0 x$ & Third-stage postpartum hemorrhage \\
\hline & $666.1 x$ & Other immediate postpartum hemorrhage \\
\hline & $666.2 x$ & Delayed and secondary postpartum hemorrhage \\
\hline & $666.3 x$ & Postpartum coagulation defects \\
\hline $\begin{array}{l}\text { Preterm rupture of } \\
\text { membranes }\end{array}$ & 658.11 & $\begin{array}{l}\text { Premature rupture of membranes, delivered, with or without } \\
\text { mention of antepartum condition }\end{array}$ \\
\hline Previous cesarean & 654.21 & Previous cesarean delivery, delivered \\
\hline \multirow[t]{2}{*}{ Severe laceration } & $664.2 x$ & Third-degree perineal laceration during delivery \\
\hline & $664.3 x$ & Fourth-degree perineal laceration during delivery \\
\hline Shoulder dystocia & $660.4 x$ & Shoulder (girdle) dystocia during labor and delivery \\
\hline \multicolumn{3}{|l|}{ Neonatal } \\
\hline \multirow[t]{2}{*}{ Abstinence syndrome } & 292.0 & Drug withdrawal \\
\hline & 779.5 & Drug withdrawal syndrome in newborn \\
\hline
\end{tabular}


Appendix A (Continued)

\begin{tabular}{|c|c|c|}
\hline Diagnosis & Code & Description \\
\hline \multicolumn{3}{|l|}{ Maternal } \\
\hline Birth defects & Numerous & $\begin{array}{l}\text { http://www.mdch.state.mi.us/pha/osr/CHI/birthdefects/ } \\
\text { BXDefectCodeGroups.html }\end{array}$ \\
\hline \multirow[t]{3}{*}{ Encephalopathy } & $348.3 x$ & Encephalopathy \\
\hline & $768.7 x$ & Hypoxic-ischemic encephalopathy \\
\hline & 779.2 & $\begin{array}{l}\text { Cerebral depression, coma, and other abnormal cerebral signs } \\
\text { in fetus or newborn }\end{array}$ \\
\hline \multirow[t]{2}{*}{ Hypoglycemia } & 251.2 & Hypoglycemia, unspecified \\
\hline & 775.6 & Neonatal hypoglycemia \\
\hline \multirow[t]{5}{*}{ Hypoxia } & $768.7 x$ & Hypoxic-ischemic encephalopathy \\
\hline & 768.9 & Unspecified birth asphyxia in liveborn infant \\
\hline & 770.12 & Meconium aspiration with respiratory symptoms \\
\hline & 770.88 & Hypoxemia of newborn \\
\hline & 775.81 & Other acidosis of newborn \\
\hline $\begin{array}{l}\text { Persistent pulmonary } \\
\text { hypertension }\end{array}$ & 747.83 & Persistent fetal circulation \\
\hline \multirow[t]{6}{*}{ Respiratory failure } & V46.11 & Dependence on respirator, status \\
\hline & 518.81 & Acute respiratory failure \\
\hline & 518.83 & Chronic respiratory failure \\
\hline & 518.84 & Acute and chronic respiratory failure \\
\hline & 770.84 & Respiratory failure of newborn \\
\hline & 770.87 & Respiratory arrest of newborn \\
\hline \multirow[t]{2}{*}{ Seizures } & $345 . x$ & Epilepsy and recurrent seizures \\
\hline & 779.0 & Convulsions in newborn \\
\hline \multirow[t]{5}{*}{ Sepsis } & 038.x & Septicemia \\
\hline & 771.81 & Septicemia of newborn \\
\hline & 785.52 & Septic shock \\
\hline & 995.91 & Sepsis \\
\hline & 995.92 & Severe sepsis \\
\hline
\end{tabular}

Abbreviation: ICD-9, International Classification of Diseases, ninth edition. 